Publicación cuatrimestral. Vol. 5, No. 4 (Número Especial), Año 2021. Pág. 23-30 CALIDAD DEL SOFTWARE APLICADO A LA EVALUACIÓN DE LA SEGURIDAD

\title{
CALIDAD DEL SOFTWARE APLICADO A LA EVALUACIÓN DE LA SEGURIDAD EN SISTEMAS DE INFORMACIÓN
}

AUTORES: $\quad$ Michelle Genesis Sinisterra Rodríguez ${ }^{1}$

Narcisa Jennifer Quimis Peñafiel ${ }^{2}$

Yaritza Paulette Gonzalez Baque ${ }^{3}$

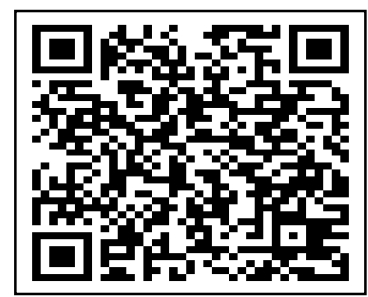

Javier Mario Merino Marcillo 4

DIRECCIÓN PARA CORRESPONDENCIA: (rodríguez-genesis9785@unesum.edu.ec)

Fecha de recepción: 03/02/2021

Fecha de aceptación: 20/05/2021

\section{RESUMEN}

Lo digital ya es parte de la vida de todos hoy en día rigiendo a empresas, entidades públicasprivadas- espacios educativos, entre otros lo que lleva a estas hacer uso de programas informáticos no siempre de buena calidad ni que brinden la seguridad que se requiere. La presente investigación tiene como objetivo hacer énfasis sobre la calidad del software y como va ligado con la seguridad de un sistema de información con el fin de ofrecer a los usuarios informáticos de acuerdo a lo investigado una perspectiva positiva que se acople a sus necesidades optimización, eficiencia y satisfacción misma que permite evaluar si un programa de computador cuenta con un nivel de calidad aceptable durante su ciclo de vida. Es importante saber que para lograr que un software sea de calidad es necesario tener en cuenta un factor significativo como lo es la seguridad, aunque esto parecería ser tarea de la administración de los sistemas, se deben utilizar ciertos mecanismos y técnicas que se encarguen de garantizar una efectiva aplicación de seguridad en los sistemas de computadoras. En este trabajo se describen las líneas de investigación y desarrollo que se realizaron en el marco de referencia hacia la calidad de software orientado a la evaluación de la seguridad en sistemas de información gracias al uso de diferentes métodos que dieron paso a realizar una excelente investigación. De acuerdo a la idea principal el modelo se espera tener un enfoque directo a la evaluación en cuestión de calidad y seguridad de software.

PALABRAS CLAVE: calidad de software; evaluación; seguridad; sistema de información.

\footnotetext{
${ }^{1}$ Estudiante de la Carrera Tecnologías de la información. Bachillerato. Noveno semestre. Estudiante de la Universidad Estatal del Sur de Manabí. Jipijapa, Manabí y Ecuador. gonzalez-yaritza6417@unesum.edu.ec

${ }^{2}$ Estudiante de la Carrera Tecnologías de la información. Bachillerato. Noveno semestre. Estudiante de la Universidad Estatal del Sur de Manabí. Jipijapa, Manabí y Ecuador.penafiel-jennifer7857@unesum.edu.ec

${ }^{3}$ Estudiante de la Carrera Tecnologías de la información. Bachillerato. Noveno semestre. Estudiante de la Universidad Estatal del Sur de Manabí. Jipijapa, Manabí y Ecuador.rodríguez-genesis9785@unesum.edu.ec
} 
González Yaritza, Peñafiel Jennifer, Rodríguez Genesis

\title{
QUALITY OF THE SOFTWARE APPLIED TO THE ASSESSMENT OF SECURITY IN INFORMATION SYSTEMS ABSTRACT
}

\begin{abstract}
The line of this research in reference to the idea subject from the birth of the software in order to offer computer users optimization, efficiency and satisfaction according to their needs, since these three characteristics allow us to evaluate if it has a level of acceptable quality during its life cycle. To achieve quality software it is necessary to take into account an important factor such as security, although this would seem to be the task of the systems administration, certain mechanisms and techniques must be used that are responsible for guaranteeing an effective application of security in computer systems. This work describes the lines of research and development that were carried out in the framework of reference towards the quality of software oriented to the evaluation of security in information systems. According to the main idea, the model must have a direct approach to the evaluation of software quality and security. Each model is made up of the same factors that are measured directly and indirectly.
\end{abstract}

KEY WORDS: evaluation; information system; security; software quality.

\section{INTRODUCCIÓN}

Es real que los avances tecnológicos en computación y software son constantes aportan un nuevo nivel de autonomía a los vehículos, los robots en los almacenes, las cámaras de seguridad y una amplia gama de servicios de internet es por ello calidad del software en un mundo globalizado toma un papel importante como un punto diferenciador, además de satisfacer las necesidades del cliente. La definición de la calidad del software según la IEEE, es "el grado con el que un sistema, componente o proceso cumple los requerimientos especificados y las necesidades o expectativas del cliente o usuario". Es necesario conocer que la calidad del software aplicado a la evaluación de la seguridad permite en gran escala controlar todo proceso además de brindar seguridad al usuario al momento de hacer uso de un sistema.

El concepto de calidad de software bajo el sustento de (Callejas Cuervo , Alarcón Aldana, \& Álvarez Carreño, 2017) se asocia a la "concordancia con los requisitos funcionales y de rendimiento explícitamente establecidos con los estándares de desarrollo plenamente documentados y con las características implícitas que se espera de todo software desarrollado profesionalmente", con base en los requisitos funcionales y no funcionales identificados en la etapa de análisis del sistema, insumo principal para implementar dichos requisitos con los atributos mínimos de calidad, fomentando la aplicación de procesos estandarizados y criterios necesarios en cada una de sus etapas, así se fomenta que el avance en el ciclo de vida del software minimice el riesgo de fracaso del proyecto. Por su parte, el Instituto de Ingenieros Eléctricos y Electrónicos (IEEE, 1990) define calidad de software como "el grado con el que un sistema, componente o proceso cumple los requerimientos especificados y las necesidades o expectativas del cliente o usuario", denotando que el énfasis radica en los requisitos específicos del sistema y en la búsqueda de la satisfacción del cliente.

Para lograr el software de calidad es necesario tener en cuenta un factor importante como lo es la seguridad, aunque esto parecería ser tarea de la administración de los sistemas, se deben utilizar ciertos mecanismos y técnicas que se encarguen de garantizar una efectiva aplicación de seguridad en los sistemas de computadoras”. El software se clasifica en básico (el sistema

24 UNESUM-Ciencias. Publicación cuatrimestral. Vol. 5, Año 2021, No. 4 (Número Especial) 
operativo) y de aplicaciones. Un sistema operativo es el alma del ordenador que sirve como medio de comunicación entre usuario y máquina, de la misma forma permite tener un control de recursos, programas y almacenamiento. El control de calidad se fomentó con el propósito de impedir que un producto defectuoso llegara al cliente y a partir de ello inicializar procesos de gestión de calidad total con el objetivo de garantizar la calidad por medio de la planificación y la creación de modelos de calidad de forma permanente. La calidad total está estrechamente relacionada con el proceso de las cinco etapas del ciclo de vida de un desarrollo de software (análisis, diseño, implementación, pruebas e implantación)

Existen varias métricas de calidad, pero las más importantes y que engloban a las demás, son sólo cuatro: corrección, facilidad de mantenimiento, integridad y facilidad de uso. Las Métricas de Calidad proporcionan una indicación de cómo se ajusta el software, a los requerimientos implícitos y explícitos del cliente. Las métricas proporcionan una indicación de la efectividad sobre las actividades en base a control y garantía de calidad.

Para garantizar la calidad de software es importante implementar algún modelo o estándar de calidad que permita la gestión de atributos en el proceso de construcción de software, teniendo en cuenta que la concordancia de los requisitos y su construcción son la base de las medidas de calidad establecidas.

\section{DESARROLLO}

¿Qué es el software?

El software es una de las herramientas de mayor utilidad en la optimización de procesos en las organizaciones, con el propósito de contar y ofrecer optimización, eficiencia y satisfacción de necesidades, razón por la cual el software debe contar con criterios que garanticen su calidad. De acuerdo con esta necesidad, diferentes entidades o investigadores han propuesto estrategias modelos, metodologías, guías, incluso normas y estándares de calidad que brindan apoyo al desarrollo y/o uso de un producto software y permiten evaluar si efectivamente tiene un nivel de calidad durante su ciclo de vida, y de esta manera fomentar un ambiente de calidad, con base en la adecuada administración de la información. (Callejas Cuervo , Alarcón Aldana, \& Álvarez Carreño, 2017)

Contextualización de calidad de software

Es de suma importancia tener un conocimiento basto sobre lo que es calidad de software sus características, modelos, métricas además de su estructura y enfoque. El software nació de la idea de ofrecer a los usuarios informáticos de acuerdo a sus necesidades optimización, eficiencia y satisfacción puesto que estas tres características nos permiten evaluar si cuenta con un nivel de calidad aceptable durante su ciclo de vida.

El software es una de las herramientas de mayor utilidad en la optimización de procesos en las organizaciones, con el propósito de contar y ofrecer optimización, eficiencia y satisfacción de necesidades, razón por la cual el software debe contar con criterios que garanticen su calidad. De acuerdo con esta necesidad, diferentes entidades o investigadores han propuesto estrategias modelos, metodologías, guías, incluso normas y estándares de calidad que brindan apoyo al desarrollo y/o uso de un producto software y permiten evaluar si efectivamente tiene un nivel de calidad durante su ciclo de vida, y de esta manera fomentar un ambiente de calidad, con base en la adecuada administración de la información.

Ciclo de vida de desarrollo de software 
Es el conjunto de etapas y estados por los que pasa desde que se plantea como necesidad o problema, por parte del cliente, hasta que se da por terminado dado como una solución completa, correcta y estable. Las principales etapas del CV son las siguientes:

Especificación de requisitos: en esta primera etapa el objetivo es conocer el problema a resolver (características, detalles, limitaciones, etc.). Una vez concluida se puede estimar el coste del proyecto.

Análisis: el lema "divide y vencerás" se hace patente en esta etapa. El problema principal se descompone en partes, obteniendo una serie de subproblemas más pequeños y abordables. En esta etapa nos centramos en el QUÉ y no en el CÓMO, esto es, identificar qué funciones realiza el sistema sin entrar en detalle.

Diseño: para los subproblemas identificados en la fase de análisis se buscan soluciones para posteriormente integrarlas en una solución global. Ahora el interés está en el CÓMO y no en el QUÉ, describiendo cada subproblema obtenido en la etapa de análisis.

Implementación: en esta etapa se codifica, según uno o varios lenguajes de programación, la solución diseñada.

Pruebas: se comprueba el funcionamiento de la aplicación respetando los requisitos y necesidades fijados con el cliente.

Instalación y mantenimiento: es la etapa de explotación del sistema, esto es, la puesta en funcionamiento en su entorno real.

Calidad de software

Básicamente se refiere al grado de desempeño de las principales características con las que debe cumplir un sistema computacional durante su ciclo de vida, dichas características de cierta manera garantizan que el cliente cuente con un sistema confiable, lo cual aumenta su satisfacción frente a la funcionalidad y eficiencia del sistema construido , además se asocia a la "concordancia con los requisitos funcionales y de rendimiento explícitamente establecidos con los estándares de desarrollo plenamente documentados y con las características implícitas que se espera de todo software desarrollado profesionalmente", con base en los requisitos funcionales y no funcionales identificados en la etapa de análisis del sistema, insumo principal para implementar dichos requisitos con los atributos mínimos de calidad, fomentando la aplicación de procesos estandarizados y criterios necesarios en cada una de sus etapas, así se fomenta que el avance en el ciclo de vida del software minimice el riesgo de fracaso del proyecto.

Para garantizar la calidad de software es importante implementar algún modelo o estándar de calidad que permita la gestión de atributos en el proceso de construcción de software, teniendo en cuenta que la concordancia de los requisitos y su construcción son la base de las medidas de calidad establecidas.

Modelos de calidad de software

Los modelos de calidad son aquellos documentos que integran la mayor parte de las mejores prácticas, proponen temas de administración en los que cada organización debe hacer énfasis, integran diferentes prácticas dirigidas a los procesos clave y permiten medir los avances en calidad. Además, identifica que la organización debe contar con un proceso que como soporte al mismo lleve una documentación, y se valga de distintas prácticas definidas en el modelo, dando apoyo a la organización para tener una mejora continua y ser más competentes, para así poder medir la calidad y brindar productor o servicios de alto nivel.

En el ámbito de la construcción de software, el modelo de calidad debe permitir evaluar el sistema, bien sea cualitativa o cuantitativamente, y de acuerdo con esta evaluación la

26 UNESUM-Ciencias. Publicación cuatrimestral. Vol. 5, Año 2021, No. 4 (Número Especial) 
organización podrá proponer e implementar estrategias que permitan la mejora del proceso dentro de las etapas de análisis, diseño, desarrollo y pruebas del software. Los modelos de calidad de software se clasifican de acuerdo con el enfoque de evaluación, ya sea a nivel de proceso, producto o calidad en uso.

Calidad a nivel de proceso

La calidad de un sistema software debe ser programada desde el inicio del proyecto, y posteriormente en cada etapa del proceso de desarrollo se debe llevar a cabo el control y seguimiento de los aspectos de calidad, para minimizar los riesgos y ofrecer soporte continuo, se garantiza así un óptimo nivel de cumplimiento de los factores de calidad, teniendo en cuenta que si en alguna de las etapas se deja de lado la verificación de los factores y criterios es posible que se presente deficiencia en alguno de éstos y disminuirá el nivel de calidad no solo del proceso, sino también del producto en desarrollo.

Calidad a nivel de producto

La principal finalidad del modelo de calidad de producto es especificar y evaluar el cumplimiento de criterios del producto, para lo cual se aplican medidas internas y/o medidas externas Este enfoque está orientado a verificar el cumplimiento de las características que permitan alcanzar la satisfacción del cliente en cuanto a los requisitos definidos en las etapas iniciales del proceso de desarrollo.

Calidad en uso

Es importante resaltar que aunque en diferentes escenarios se utilizan los términos usabilidad y calidad en uso, con el mismo propósito y de forma intercambiable tienen significados distintos, principalmente porque el concepto de calidad en uso es más amplio y abarca más elementos que la usabilidad, y esta última es una de las características de calidad de un producto software, la calidad en uso se define como el "conjunto de atributos relacionados con la aceptación por parte del usuario final y seguridad", y está basada en la eficacia, productividad, seguridad y satisfacción.(Álvarez, 2016)

Calidad de los sistemas de información

Es un modelo que trasforma datos en información organizada, significativa y útil para la toma de decisiones efectivas para los gerentes, la calidad del software es el conjunto de cualidades que lo caracterizan y que determinan su utilidad y existencia además es la concordancia con los requerimientos funcionales y de rendimientos explícitamente establecidos, con los estándares de desarrollo y con la característica implícitas que se espera de todo software.

Métricas de calidad de sistemas de información

Las métricas son escalas de unidades sobre las cuales puede medirse un atributo cuantificable, en software se debe recopilar y analizar datos basándose en mediciones reales de software, los atributos son características observables del producto o del proceso de software.

Las métricas de calidad de sistema de información se utilizan para poder evaluar y controlar el proceso de desarrollo y software de forma que les permita indicar la calidad del producto, evaluar la productividad de los desarrolladores. (Tomalá, 2009)

\section{Seguridad de los sistemas de información}

Cuando hablamos de seguridad de la información estamos indicando que dicha información tiene una relevancia especial en un contexto determinado y que, por tanto, hay que proteger. La Seguridad de la Información se puede definir como conjunto de medidas técnicas, organizativas y 
legales que permiten a la organización asegurar la confidencialidad, integridad y disponibilidad de su sistema de información.

Hasta la aparición y difusión del uso de los sistemas informáticos, toda la información de interés de una organización se guardaba en papel y se almacenaba en grandes cantidades de abultados archivadores. Datos de los clientes o proveedores de la organización, o de los empleados quedaban registrados en papel, con todos los problemas que luego acarreaba su almacenaje, transporte, acceso y procesado.

Los sistemas informáticos permiten la digitalización de todo este volumen de información reduciendo el espacio ocupado, pero, sobre todo, facilitando su análisis y procesado. Se gana en 'espacio', acceso, rapidez en el procesado de dicha información y mejoras en la presentación de dicha información. Pero aparecen otros problemas ligados a esas facilidades. Si es más fácil transportar la información también hay más posibilidades de que desaparezca 'por el camino'. Si es fácil acceder a ella también es fácil modificar su contenido, etc.

Desde la aparición de los grandes sistemas aislados hasta nuestros días, en los que el trabajo en red es lo habitual, los problemas derivados de la seguridad de la información han ido también cambiando, evolucionando, pero están ahí y las soluciones han tenido que ir adaptándose a los nuevos requerimientos técnicos. Aumenta la sofisticación en el ataque y ello aumenta la complejidad de la solución, pero la esencia es la misma.

Existen también diferentes definiciones del término Seguridad Informática. De ellas nos quedamos con la definición ofrecida por el estándar para la seguridad de la información ISO/IEC 27001, que fue aprobado y publicado en octubre de 2005 por la International Organization for Standardization (ISO) y por la comisión International ElectrotechnicalCommission (IEC). La seguridad informática consiste en la implantación de un conjunto de medidas técnicas destinadas a preservar la confidencialidad, la integridad y la disponibilidad de la información, pudiendo, además, abarcar otras propiedades, como la autenticidad, la responsabilidad, la fiabilidad y el no repudio. (Gerencial, 2016)

Un sistema será seguro o fiable con la ayuda de 4 aspectos:

- Integridad: Es el atributo por el cual la información se Considera completa y correcta, se alcanza con un apropiado diseño de sistemas y procedimientos, el entrenamiento del personal, una supervisión efectiva y controles eficientes. Los datos tienen integridad cuando están libres de errores intencionales o no intencionales (en grado necesario para operaciones normales) y cuando no han sido modificados ni procesados en forma no autorizada.

- Confidencialidad: Es la condición de la información por la cual ésta se encuentra protegida contra la divulgación indebida.

- Privacidad: Es la condición de la información por la cual ésta se halla protegida contra la utilización, la observación o la divulgación maliciosas y no autorizadas de datos sobre las personas. Con el mismo significado, se usa a veces el término "intimidad". La privacidad alude a la información que un individuo o una empresa no desea que tenga difusión generalizada. Teniendo en cuenta la privacidad, debe definirse cuidadosamente qué información será recogida, cómo y quién la utilizará, y la forma en que será revisada, modificada y corregida. El conflicto se produce cuando los derechos de los individuos se contraponen con las necesidades de las organizaciones privadas o públicas.

- Continuidad: Se refiere a la posibilidad de seguir operando normalmente a pesar de los daños que se hubieran ocasionado. Según el tipo de operaciones, esta continuidad será más o menos crítica.

28 UNESUM-Ciencias. Publicación cuatrimestral. Vol. 5, Año 2021, No. 4 (Número Especial) 


\section{Los peligros pueden tener los sistemas de información}

Ambientales naturales: Inundación, incendio, filtraciones, alta temperatura, terremoto, derrumbe, explosión, corte de energía, disturbios, etc.

Ambientales operativas: Caída o falla de: procesador, periféricos, comunicaciones, software de base o de aplicación, aire acondicionado, sistema eléctrico, etc.

Humanas no intencionales: Errores y omisiones en el ingreso de datos, en la operación, en el uso de archivos o programas, en el desarrollo de sistemas o en el uso de respaldo (backup). Además, pérdida de soportes, falta de documentación actualizada, accidentes en la prueba de programas, daño accidental de archivos.

Humanas intencionales: Fraude (hurto, robo, defraudación o uso indebido de recursos),daño intencional (virus, terrorismo, vandalismo, sabotaje,, programación maliciosa o infiltración en líneas), invasión a la privacidad (Salvatierra, s.f.)

Materiales Y Métodos

La propuesta constituye una metodología integral para la identificación de la evaluación de la calidad de software para sistemas de información, teniendo en cuenta que La tecnología hoy en día es una herramienta imprescindible para el desarrollo de factores económicos, culturales y sociales, por lo cual es importante crear herramientas tecnológicas que agreguen valor a las actividades cotidianas de las personas, facilitando su uso y garantizando su correcto funcionamiento cobra más importancia la evaluación de la calidad del software, entre los métodos utilizados tenemos:

Histórico - Lógico

Este método fue utilizado en la construcción de todo el proyecto, para determinar la base teórica sobre el análisis de la calidad del software aplicado a la evaluación de la seguridad en sistemas de información.

Análisis - Síntesis

Este método fue empleado para realizar un análisis de la información en torno al proceso de la calidad del software aplicado a la evaluación de la seguridad en sistemas de información, en base a ello evidenciar los requisitos más característicos para saber qué se hace uso de un software de calidad.

Bibliográfico

Fue utilizado para sustentar el desarrollo de la presente investigación en base a los contenidos consultados y sistematizados, para de esa manera delinear acciones para la propuesta de un prototipo basado en el objeto de estudio.

Discusión De Los Resultados

Según lo establece (25010, 2011), el ámbito de aplicación de los modelos de calidad incluye la especificación y evaluación de soporte de software y sistemas informáticos intensivos en software desde diferentes perspectivas por parte de aquellos asociados con su adquisición, requisitos, desarrollo, uso, evaluación, soporte, mantenimiento, aseguramiento y control de calidad y auditoría. Los modelos pueden, por ejemplo, ser utilizados por desarrolladores, adquirentes, personal de control y garantía de calidad y evaluadores independientes, en particular los responsables de especificar y evaluar la calidad del producto de software. Además de que la seguridad se define como la "Capacidad de protección de la información y los datos de manera 
que personas o sistemas no autorizados no puedan leerlos o modificarlos”, y, considerando las subcaracterísticas esperables o deseables para un producto software en cuanto a seguridad y calidad, de acuerdo a lo tratado en esta línea de investigación consiste de las siguientes características:

- Identificar los requisitos de software y sistema.

- Validar la exhaustividad de una definición de requisitos.

- Identificar los objetivos de diseño de software y sistemas.

- Identificar los objetivos de las pruebas de software y sistemas.

- Identificar los criterios de control de calidad como parte del aseguramiento de la calidad.

- Confidencialidad. Capacidad de protección contra el acceso de datos e información no autorizados, ya sea accidental o deliberadamente.

- Integridad. Capacidad del sistema o componente para prevenir accesos o modificaciones no autorizados a datos o programas de ordenador.

- No repudio. Capacidad de demostrar las acciones o eventos que han tenido lugar, de manera que dichas acciones o eventos no puedan ser repudiados posteriormente.

- Responsabilidad. Capacidad de rastrear de forma inequívoca las acciones de una entidad.

- Autenticidad. Capacidad de demostrar la identidad de un sujeto o un recurso.

\section{CONCLUSION}

Los modelos de calidad básicos han sido la base para que los modelos actuales sean más complejos en la evolución del software, para así optimizar los procesos de las organizaciones y garantizar que se cumple con criterios o estándares que respaldan la calidad de la gestión de la seguridad.

En su mayoría, la implementación de modelos de calidad de software ha sido adoptada por empresas desarrolladoras de software, sin embargo, algunos modelos permiten adaptarse a contextos empresariales con fines diferentes al del desarrollo o construcción de software.

\section{REFERENCIASBIBLIOGRÁFICAS}

25010, I. I. (2011). iso. Obtenido de www.iso.org: https://www.iso.org/standard/35733.html

$\begin{array}{lllllll}\text { Álvarez, } & \text { M. } & \text { C. } & \text { (08 de } & \text { Octubre } & \text { de } & \text { 2016). }\end{array}$ https://www.redalyc.org/jatsRepo/2654/265452747018/html/index.html

Callejas Cuervo , M., Alarcón Aldana, A. C., \& Álvarez Carreño, A. M. (2017). scielo. Obtenido de www.scielo.org.co: http://www.scielo.org.co/pdf/entra/v13n1/1900-3803-entra-13-01-00236.pdf

Gerencial, S. d. (10 de Febrero de 2016). Obtenido de SEGURIDAD EN LOS SISTEMAS DE INFORMACION: https://sistemasinformacionsite.wordpress.com/2016/02/10/seguridad-en-los-sistemas-de-informacion/

Salvatierra, M. A. (s.f.). Obtenido de La seguridad de los sistemas de información: https://www.monografias.com/trabajos103/seguridad-sistemas-informacion/seguridad-sistemasinformacion.shtml

Tomalá, S. C. (2009). Metricas de calidad de los sistemas de información . Obtenido de https://www.dspace.espol.edu.ec/bitstream/123456789/4908/1/7708.pdf

30 UNESUM-Ciencias. Publicación cuatrimestral. Vol. 5, Año 2021, No. 4 (Número Especial) 